\title{
Theoretical modeling of interstitial carbon impurities in paramagnetic Fe-Mn alloys
}

\author{
A. V. Ponomareva $\odot,{ }^{1}$ B. O. Mukhamedov $\odot,,^{1,2}$ and I. A. Abrikosov $\circledast^{1,3}$ \\ ${ }^{1}$ Materials Modeling and Development Laboratory, National University of Science and Technology “MISIS”, Moscow 119049, Russia \\ ${ }^{2}$ Department of System and Software Engineering, National University of Science and Technology “MISIS”, Moscow 119049, Russia \\ ${ }^{3}$ Department of Physics, Chemistry, and Biology (IFM), Linköping University, Linköping SE-581 83, Sweden
}

(Received 16 August 2019; revised manuscript received 15 November 2019; accepted 7 January 2020; published 4 February 2020)

\begin{abstract}
We present a generalization of a model that takes into account the magnetic disorder of paramagnetic host with interstitial point defects towards the case of alloy hosts. In the framework of disordered local moment approach combined with magnetic sampling method, we calculate solution enthalpy of carbon impurity in the paramagnetic fcc Fe-Mn steels. First, we use the magnetic special quasirandom structure (M-SQS) method for simulation of the paramagnetic state in Fe-Mn alloys without impurity. Here, Fe and $\mathrm{Mn}$ atoms are randomly distributed at the sites of a supercell following the chemical SQS method. Next, to calculate the energies for various magnetic realizations around the interstitial carbon impurity, we vary the position of the impurity within the SQS. Our calculations show that in alloys containing $\sim 20$ at. \% Mn, the solution enthalpy of carbon reduces compared to the pure fcc-Fe. By analyzing the local and global effects of impurity on the properties of the matrix, we discuss various factors that could increase the carbon solubility in high-manganese austenitic steels.
\end{abstract}

DOI: 10.1103/PhysRevMaterials.4.024401

\section{INTRODUCTION}

High-manganese austenitic steels (so-called TRIP and TWIP steels) are widely used in manufacturing of tools with extremely high strength. These steels are of great interest because of their unique combination of high strength and plasticity, great ductility and energy absorption during the deformation, as well as due to their lower weight compared to carbon steels. As a rule, the high-manganese steels contain 10-35 wt. \% of manganese, and the concentration of carbon can reach up to $1.5 \mathrm{wt}$. \%. In order to improve some specific properties, the Fe-Mn alloys may also contain other elements, like aluminum, copper, titanium, tungsten, molybdenum, chromium, etc. Carbon in these steels strongly influences the mechanical properties, especially during martensitic transformations. According to experimental data [1], in the Fe-22Mn-C austenite steels the product of deformation changes from mechanical twins (TWIP) to mechanical $\varepsilon$ martensite (TRIP) if the carbon concentration reduces from 0.6 to 0.2 wt. \%. Therefore, knowledge about thermodynamics of the carbon solution into Fe-Mn austenite is highly important for improvement and optimization of mechanical properties of steels. There were several experimental studies [2,3] where the authors reported higher temperature of martensitic transformation in Fe-Mn steels than the Néel temperature of the steels. This means that the martensitic transformations occur in the paramagnetic austenite. However, most of the ab initio calculations [4-8] still focus on antiferromagnetic Fe-Mn-based austenite to make predictions on martensitic transformations. Therefore, simulations of the paramagnetic state of Fe-Mn steels is a highly relevant task. Due to the importance of carbon in these steels, the main purpose of our work is to study the solution thermodynamics of carbon in paramagnetic Fe-Mn steels and to analyze the local and global effects induced by the interstitial $\mathrm{C}$ impurities on the properties of the material.

From a theoretical point of view, the existence of local magnetic moments in the high-temperature paramagnetic state of Fe-Mn steels makes it quite challenging to study the processes of alloying and formation of point defects. There are a number of first-principle approaches to describe the paramagnetic state of magnetic materials. Perhaps the most adequate approximation at present is the dynamic mean field theory (DMFT) [9]. However, it is well known that the incorporated defects reduce the symmetry of the lattice, which makes DMFT calculations quite time consuming. On the other hand, the simulation of this chemically complex paramagnetic system within the model of disordered local moments (DLM) [9-14] combines high efficiency with sufficient accuracy. Until recently, the DLM approximation was realized in the framework of the coherent potential approximation (CPA) [15] and was not suitable for simulations of point defects inducing sufficiently strong local distortions of the crystal lattice, like interstitial impurities. However, in Ref. [16], Alling et al. successfully combined the DLM picture and the supercell method, introducing two differerent techniques, the magnetic special quasirandom structure technique (SQS) [17] method and the magnetic sampling method (MSM) [16]. The reliability of these methods was demonstrated in simulations of $\mathrm{CrN}$ compound. It was found that the thermodynamic properties of $\mathrm{CrN}$ calculated within DLM method are in good agreement with the results obtained by DLM in combination with the molecular dynamics (DLM-MD) [18] method, which takes into account the lattice dynamics of the paramagnetic system. Later Ponomareva et al. [19] combined the MSM method with magnetic SQS method to simulate the paramagnetic state of magnetic solids with point defects. This 
combination allows one to perform accurate and computationally efficient calculations of solution enthalpy $H_{\text {sol }}$ for interstitial and substitutional impurities, e.g., in Fe-austenite [19]. In the present work, we generalize this technique to the case of an alloy host with interstitial point defects and perform calculations of solution enthalpy for carbon impurity in the paramagnetic Fe-Mn alloys.

\section{CALCUlation METHOD}

\section{A. Computational details}

Calculations are performed in the framework of the DFT using a projector-augmented wave method implemented in the VASP code [20-23]. The exchange-correlation effects were treated using the generalized gradient approximation [24]. The cutoff energy for plane waves was set to $500 \mathrm{eV}$. The convergence criterion for electronic subsystem was chosen to be equal to $10^{-4} \mathrm{eV}$ for subsequent iterations. The relaxation of atomic positions was carried out by calculating the HellmanFeynman forces [25] and the stress tensor and using them in the conjugated gradient method. Relaxation was considered to be completed when the forces on the ions became of the order of $10^{-3} \mathrm{eV} / \AA$. The volume and shape of the cell were fixed during iterations. The integration over the irreducible part of Brillouin zone was carried out using the Monkhorst-Pack method [26] on a grid of $2 \times 2 \times 2 k$ points. We checked that in the case of $4 \times 4 \times 4 k$-point mesh the solution enthalpy differs by less than $0.002 \mathrm{eV}$. The carbon atoms were located in octahedral interstitial sites. Calculations were performed for periodic supercells with $108\left(\left(\left\{\mathrm{Fe}_{43} \mathrm{Mn}_{11}\right\} \uparrow\left\{\mathrm{Fe}_{43} \mathrm{Mn}_{11}\right\} \downarrow\right)\right.$ and $109\left(\left\{\mathrm{Fe}_{43} \mathrm{Mn}_{11}\right\} \uparrow\left\{\mathrm{Fe}_{43} \mathrm{Mn}_{11}\right\} \downarrow \mathrm{C}_{1}\right)$ ions.

\section{B. Modeling of magnetic and chemical disorder}

For simulation of a substitutional alloy with isolated interstitial impurity, we generalize the theoretical approach introduced in Ref. [19] to be able to describe the chemical and magnetic disorder in matrix simultaneously. Our computational scheme has the following steps. First, we use chemical and magnetic SQS methods for simulation of the paramagnetic state of Fe-Mn alloys without any interstitial impurity. Herewith, the magnetic and chemical disorder in the studied system is simulated as a four-component system $\left(\left\{\mathrm{Fe}_{x / 2} \mathrm{Mn}_{(1-x) / 2}\right\} \uparrow\left\{\mathrm{Fe}_{x / 2} \mathrm{Mn}_{(1-x) / 2}\right\} \downarrow\right)$, which describes the host solid solutions. Here, $\mathrm{Fe}$ and $\mathrm{Mn}$ atoms are randomly distributed; and their local magnetic moments are collinear but randomly oriented as well. Note that for simulation of pure iron, we considered a two-component system with only magnetic disorder. Next, we realize that during the long timescale associated with a diffusion process of interstitial impurity dissolution, the magnetic moments of atoms in the host alloy change their orientations many times. Thus, there are constant variations of magnetic configurations in the $\mathrm{C}$ impurity surrounding. Moreover, as we model the high-temperature paramagnetic state, the diffusion is relatively fast, and the impurity may be present in different chemical surroundings. To catch the dynamic behavior of the magnetic system and the chemical complexity of the studied system, we need to approximate the paramagnetic state of the alloy by considering a set of different magnetic and chemical configurations, and then average calculated properties over the set. In other words, we need to calculate the energies for various magnetic and chemical realizations. We achieve this by varying the impurity positions within the SQS, placing the impurity at different interstitial sites of the considered supercell. For simulation of fcc Fe we carried out calculations for 40-50 impurity positions on the various interstitial sites of magnetic SQS until we achieved the convergence of the impurity solution energy with cumulative average. For Fe-Mn alloys we considered 50-70 impurity positions. Note that the average energy represents the potential energy of the alloy that contains the single impurity (the concentration of impurities is determined by the size of the SQS). Generally speaking, the potential energy should be determined by averaging over the Boltzmann distribution at a certain temperature, where one should use the energies obtained with and without impurities. However, in Ref. [19] it was shown that for the fcc Fe-C system the averaging over the Boltzmann distribution and the arithmetic averaging methods give results that differ by less than $0.04 \mathrm{eV}$ at temperatures corresponding to the stability field of fcc Fe. Therefore, in the present work we used the arithmetic average of the energies obtained with and without impurities over MSM-SQS configurations in all the calculations. All other details of the averaging are carefully described in Ref. [19].

Thus, in our approach the solution enthalpy of carbon is determined as

$$
H_{\mathrm{sol}}=\frac{\partial \Delta H_{\mathrm{f}}}{\partial x},
$$

where $\Delta H_{f}=\left\langle E\left\{(\mathrm{Fe}-\mathrm{Mn})_{1-x} C_{x}\right\}\right\rangle-(1-x)\langle E\{\mathrm{Fe}-\mathrm{Mn}\}\rangle$ $-x E\{C\}$ is the formation enthalpy of the alloy averaged over all the considered positions of the interstitial impurity in the SQS.

We define the solution enthalpy with the reference to carbon (graphite) and fcc Fe-Mn alloy:

$$
\begin{aligned}
\left\langle H_{\mathrm{sol}}\right\rangle= & (N+1)\left\langle E\left((\mathrm{Fe}-\mathrm{Mn})_{1-x} C_{x}\right)\right\rangle \\
& -N\langle E(\mathrm{Fe}-\mathrm{Mn})\rangle-E(C),
\end{aligned}
$$

where $\mathrm{N}$ denotes the number of atoms in the matrix (without impurities).

Further, to check the validity of our technique, we performed the calculations for two different sets of magnetic and chemical realizations. In other words, two different SQS were used for simulation of the matrix. As we demonstrate below, the results calculated for both the SQS are very close to each other.

Following the scheme successfully applied in Ref. [19], we carried out the total energy calculations using experimental values of the lattice parameters. At the same time, the average pressure for the cells with impurities was kept equal to the pressure of the cell without impurity. Detailed information on chosen lattice parameters is given in the Supplemental Material [27].

The proposed scheme is valid at temperatures well above the magnetic transition temperature, therefore, we can neglect the short-range magnetic effects. Since the magnetic transition temperature of fcc Fe-Mn alloys is about the room temperature [2,3], the DLM picture is adequate for our calculations. Moreover, as will be shown in Sec. III B, the energy variations between different magnetic configurations 
TABLE I. Theoretical and experimental values of solution enthalpy $\left(\left\langle H_{\text {sol }}\right\rangle\right)$ of $\mathrm{C}$ in the paramagnetic fcc $\mathrm{Fe}$ and $\mathrm{Fe}_{79.6} \mathrm{Mn}_{20.4}$ alloys (in eV).

\begin{tabular}{|c|c|c|}
\hline & Theory, MSM-SQS & Experiment \\
\hline \multirow{5}{*}{$\mathrm{Fe}$} & \multirow{5}{*}{$0.20[19]$} & $0.36[31]$ \\
\hline & & $0.40[32]$ \\
\hline & & $0.41[33]$ \\
\hline & & $0.43[34]$ \\
\hline & & $0.46[35]$ \\
\hline \multirow{2}{*}{$\mathrm{Fe}_{79.6} \mathrm{Mn}_{20.4}$} & SQS $1 \quad 0.12$ & \multirow{2}{*}{$0.37[35]$} \\
\hline & SQS $2 \quad 0.10$ & \\
\hline
\end{tabular}

( $\sim 10 \mathrm{meV} /$ supercell) are much smaller than the value of $k_{B} T$ ( $\sim 100 \mathrm{meV}$ at $T \sim 1200 \mathrm{~K}$ ), which justifies the use of the DLM picture, as well as the MSM-SQS approach.

It is well known that at high temperatures the effects of lattice vibrations, including the thermal expansion, become increasingly important. For a more accurate description of the entropic effects in a magnetic system above the magnetic transition temperature one would have to use, for example, the DLM-MD technique combined with the temperature dependent effective potential method [28], or a combination of molecular and spin dynamics [29]. However, such calculations would be really time consuming, while the proposed technique allows one to obtain a quick and reliable estimation of the impurity solution enthalpies within the validity limits of the DLM picture. Given the size of the studied systems, the contributions due to lattice vibrations have not been considered explicitly in this work. However, they have been included, at least partly, via the thermal expansion of Fe-based solid solution, as discussed in the Supplemental Material.

\section{RESULTS}

\section{A. Solution enthalpy of carbon into paramagnetic fcc Fe and Fe-Mn alloys}

Using the SQS-MSM method and Eq. (2), we have calculated the solution enthalpies $\left\langle H_{\text {sol }}\right\rangle$ of carbon impurity in paramagnetic Fe-Mn alloys. The results are presented in Table I. The theoretical $\left\langle H_{\text {sol }}\right\rangle$ for pure Fe are taken from Ref. [19]. As the reference states, we considered DLM fcc structure for Fe and Fe-Mn alloys; and for carbon we use the graphite structure with experimental values of lattice parameters $(a=2.46 \AA$, $c=6.65 \AA$ ) [30].

From Table I we see that the solution enthalpy of $\mathrm{C}$ in the alloy containing $\sim 20$ at. $\%$ of $\mathrm{Mn}$ is lower compared to pure paramagnetic $\gamma$ iron. Note, that the present values of $\left\langle H_{\text {sol }}\right\rangle$ for paramagnetic Fe were obtained for supercells without the so-called spin-flip transition (SFT). In $\gamma$ iron the SFT is induced by impurity and represents the reorientation of $\mathrm{Fe}$ local magnetic moment relative to primary direction inside the matrix without impurity. In our previous work [19] we showed that nearly $40 \%$ of magnetic realizations of the paramagnetic binary Fe-C alloy had SFT. The spin-flip takes place on the atoms located in first coordination shell (CS) of impurity atom [19]. This fact indicates that carbon impurity increases the local magnetic moment of the nearest neighbor (NN) cluster; and the first CS becomes strongly polarized. The magnetic realizations induced by the SFT (e.g., with strong polarization) exhibit a lower local solution enthalpy of carbon compared to weakly polarized cells. Thus, accounting for the SFT the $\left\langle H_{\text {sol }}\right\rangle$ is equal to $0.2 \mathrm{eV} \mathrm{[19].} \mathrm{For} \mathrm{overcooled}$ austenite, the statistical weight of these polarizations might be important; but at high temperatures the amount of these configurations is most probably low. Therefore, for the hightemperature regions we did not consider them in calculations of solution enthalpy, and as the result we got $\left\langle H_{\text {sol }}\right\rangle=0.27 \mathrm{eV}$. The point is that in the studied $\mathrm{Fe}_{79.6} \mathrm{Mn}_{20.4}$ alloy we did not observe the similar effect (we will discuss this in more detail below), and therefore consider it irrelevant for the comparison between the solution enthalpies of $\mathrm{C}$ in the pure fcc Fe and in the alloy. The obtained values of $\left\langle H_{\text {sol }}\right\rangle$ are 0.12 and $0.10 \mathrm{eV}$ for two sets of SQS, which puts the theoretically predicted decrease of solution enthalpy of $\mathrm{C}$ between elemental host and the alloy in quantitative agreement with experiment [35]. We emphasize that the difference between the theory and experiment in absolute values may appear as high. But in fact, it is not. Typical solution enthalpies are of the order of several $\mathrm{eV}$ and can be calculated from first principles with the similar absolute accuracy $(0.1$ to $0.2 \mathrm{eV})$ as in our case [36]. The magnitudes of the solution enthalpy of $\mathrm{C}$ in fcc Fe and Fe-Mn alloy are just too low. Of course, there are several reasons that still affect calculated accuracy, including limited size of supercells, which does not catch the long-wave magnetic interactions in fcc Fe [37], neglect of lattice vibrations, as well as the effects of strong electronic correlations and longitudinal spin fluctuations.

\section{B. Local and global effects of impurity on matrix properties}

It is important to underline that the proposed technique makes it possible to analyze average values of global properties (like $\left.\left\langle H_{\text {sol }}\right\rangle\right)$, as well as local properties. Let us first consider the effect of impurity on the local distortions of underlying crystal lattices for $\mathrm{Fe}-\mathrm{C}$ and $\mathrm{Fe}-\mathrm{Mn}-\mathrm{C}$ alloys. Figure 1 illustrates the average relative displacements with regard to the ideal positions $\bar{R}^{i}(\%)$ of matrix atoms for the first five coordination spheres around the interstitial impurity. The average distortions are defined as

$$
\bar{R}^{i}=\frac{1}{n} \sum_{\sigma=1}^{n} \frac{1}{m_{i}} \sum_{l=1}^{m_{i}} \frac{\left(r_{l}^{i}-r_{\text {ideal }}^{i}\right)}{r_{\text {ideal }}^{i}}
$$

where $r_{l}^{i}$ is a distance between $\mathrm{C}$ atom and a matrix atom located at $l$-th position in $i$-th coordination sphere of $\mathrm{C}$ in fully relaxed supercell; $r_{\text {ideal }}^{i}$ is the same distance for ideal lattice; $m_{i}$ is total number of the matrix atoms in $i$-th coordination sphere. In Eq. (3) the inner sum runs over $i$-th CS and the outer sum runs over $n$ number of $\sigma$-magnetic configurations of MSM-SQS.

From Fig. 1 we see that incorporation of carbon induces significant dilatations of the lattice, which rapidly decrease from a maximum value for the first CS to almost zero for the fifth CS. In Fe-Mn-C alloys, the average stretching of $\mathrm{Fe}-\mathrm{C}$ bond on the first $\mathrm{CS}$ are close to 5\% (SQS 1) and $5.1 \%$ (SQS 2), while for binary Fe-C alloy it is about $\sim 5.8 \%$. The 


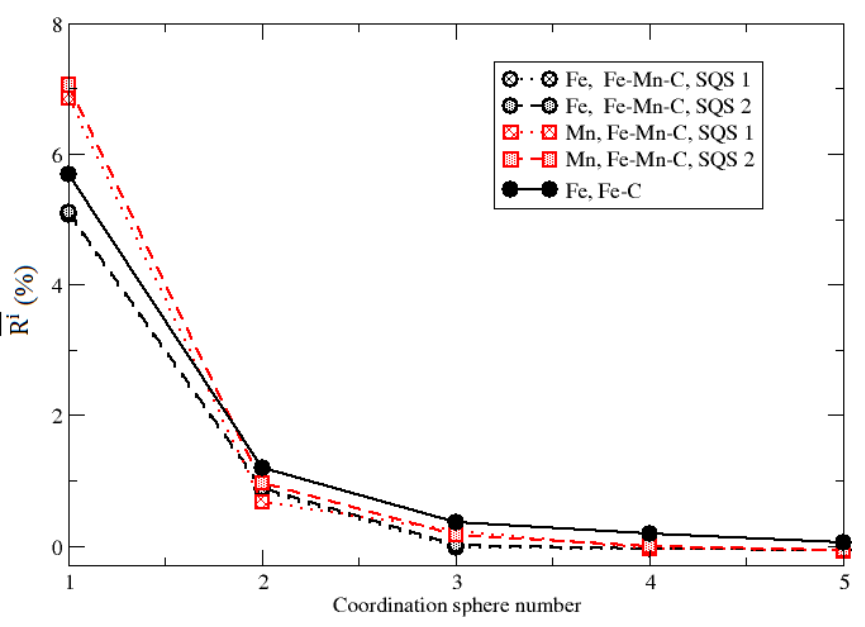

FIG. 1. Average relative displacements $\bar{R}^{i}(\%)$ of matrix atoms for the first five coordination spheres around carbon interstitial atom. The Fe-C results were taken from Ref. [19].

weakening of dilatations is due to an increase of the alloy lattice parameter from $3.64 \AA$ [38] for $\mathrm{Fe}_{99.1} \mathrm{C}_{0.9}$ to $3.658 \AA$ for $\mathrm{Fe}_{78.9} \mathrm{Mn}_{20.2} \mathrm{C}_{0.9}$ (see Supplemental Material). In Fe-Mn-C alloy, the value of $\bar{R}^{1}$ around $\mathrm{Mn}$ atoms is larger compared to that for Fe atoms and amounts to $\sim 7 \%$. Note that in Fe-Mn-C alloys the values of deformation for both supercells, SQS 1 and SQS 2 are very close to each other. From Fig. 1 one sees that for Fe-Mn-C the deformation in the second coordination shell is smaller compared to the binary $\mathrm{Fe}-\mathrm{C}$ alloys, even around $\mathrm{Mn}$ atom, where the stretching is 1.5 times higher than around $\mathrm{Fe}$ atom.

Now let us consider the influence of interstitial atoms on the magnetic properties of the matrix. As can be seen from Fig. 2, in the first coordination sphere of $\mathrm{C}$ impurity, the local magnetic moments on $\mathrm{Fe}$ and $\mathrm{Mn}$ atoms become significantly smaller compared to their values in the matrix. The average values of local magnetic moments on $\mathrm{Mn}$ atoms are higher compared to $\mathrm{Fe}$ atoms. In the binary $\mathrm{Fe}-\mathrm{C}$ alloy the average

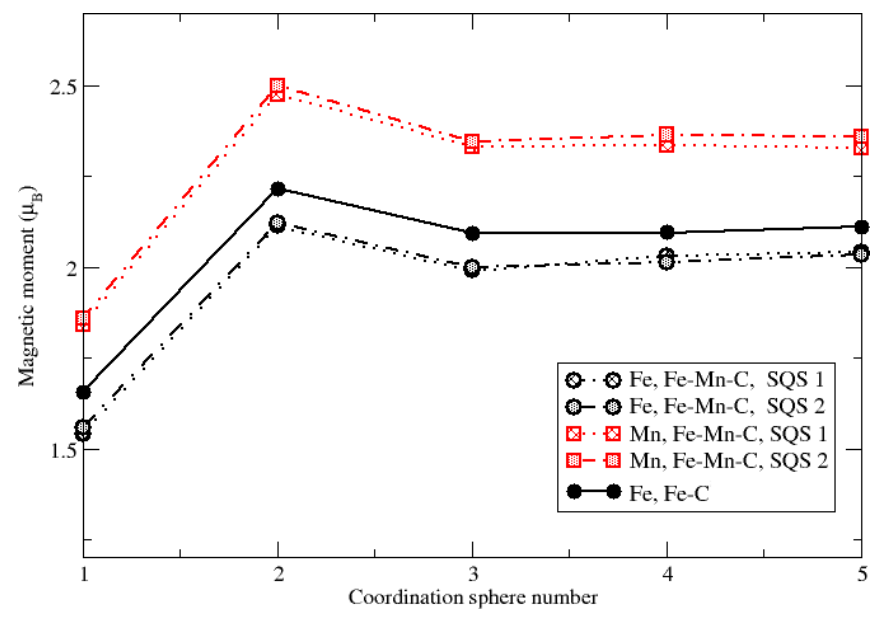

FIG. 2. Calculated average magnetic moments on $\mathrm{Fe}$ and $\mathrm{Mn}$ atoms in paramagnetic fcc Fe-Mn alloys as a function of number of coordination shell around $\mathrm{C}$ impurity. Results for Fe-C alloy from Ref. [19] are shown for comparison.

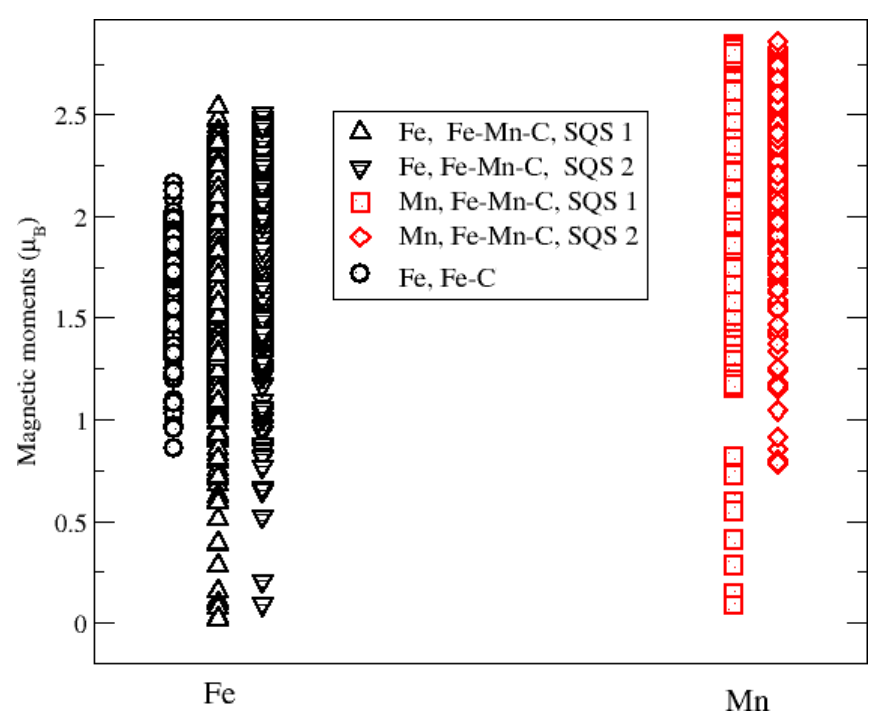

FIG. 3. Calculated local magnetic moments of all $\mathrm{Fe}$ and $\mathrm{Mn}$ atoms in the NN of impurity. The Fe-C results were taken from Ref. [19].

magnetic moment on $\mathrm{Fe}$ atoms is higher in comparison to the $\mathrm{Fe}-\mathrm{Mn}-\mathrm{C}$ alloy even though the multicomponent alloys have larger lattice parameters. This is so because of the significantly low values of magnetic moments $\sim 0-0.5 \mu_{\mathrm{B}}$ (see Fig. 3) on the NN atoms of the C impurity in the Fe-Mn-C alloys. In Ref. [19] it was shown that the low values of $\mathrm{Fe}$ magnetic moments in $\mathrm{Fe}-\mathrm{C}$ alloy can be observed only for ideal (unrelaxed) lattice. After the relaxation, all the local magnetic moments increase above $0.5 \mu_{\mathrm{B}}$.

On the contrary, in Fe-Mn-C alloys, the presence of magnetic moments with small values can be explained by the fact that it is impossible to satisfy the signs of exchange pair interactions for some of $\mathrm{Fe}-\mathrm{Fe}, \mathrm{Fe}-\mathrm{Mn}$ and $\mathrm{Mn}-\mathrm{Mn}$ pairs in the nearest neighborhood of the $\mathrm{C}$ impurity, as will be discussed in more detail below. Consequently, the system reacts by decreasing, and even quenching the local magnetic moments on the NN atoms. In addition, Fig. 2 shows that screening of the interstitial impurity is quite effective, and the perturbation of magnetic moments becomes almost negligible starting from the third coordination shell.

In all the studied alloys, like in $\gamma$-Fe, the magnitude of magnetic moment induced on carbon increases with the magnetization of the first CS of the impurity and becomes equal to $\pm 0.15 \mu_{\mathrm{B}}$ for the highest NN magnetization (see Fig. 4). Note that the magnetization of the $\mathrm{NN}$ is calculated as a sum of magnetic moments of the matrix atoms $\sum \mu^{\mathrm{NN}}$ with accounting for their signs. If the first CS consists of three spin-up atoms and three spin-down atoms the total $\sum \mu^{\mathrm{NN}}$ will be close to zero. In the opposite limit, i.e., for the ferromagnetic alignment of local moments in the first $\mathrm{CS}$, the sum $\sum \mu^{\mathrm{NN}}$ is in the range of $12-15 \mu_{\mathrm{B}}$. Note that the magnetic moment, induced on the $\mathrm{C}$ impurity, is related to the strong hybridization between $p_{z}$ electrons of carbon and valence $3 d$ electrons of $\mathrm{Fe}$ [39], and it is oriented antiparallel to the total magnetic moment of atoms surrounding carbon in the nearest neighborhood.

Figure 5 shows the total energies of all studied MSM realizations as functions of the total magnetic moment of $\mathrm{Fe}$ 


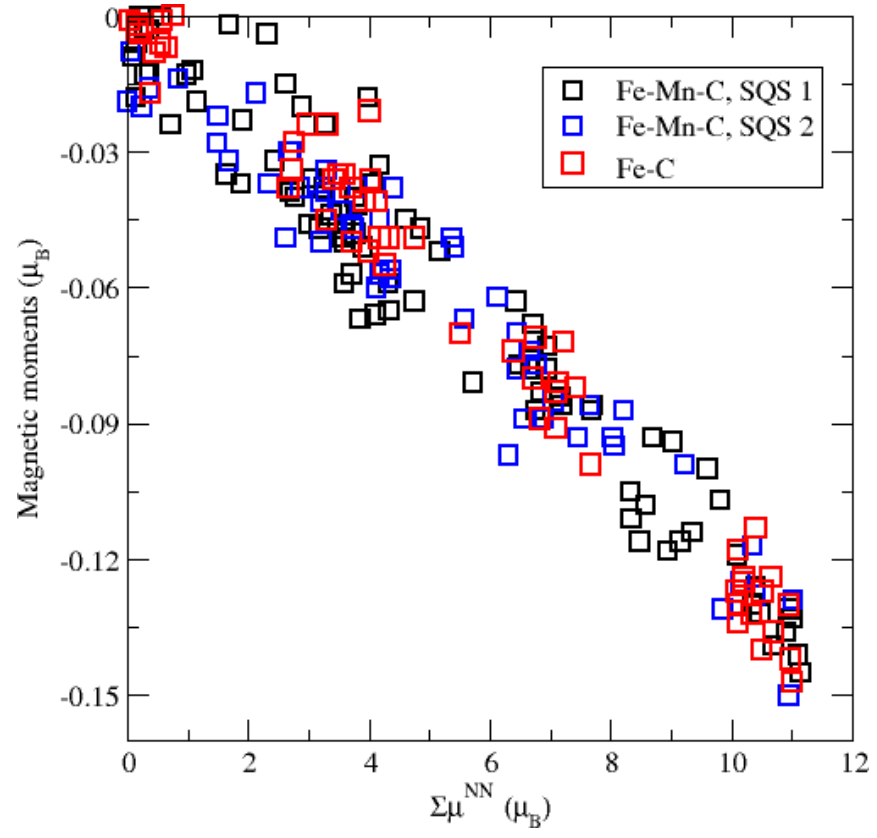

FIG. 4. Induced magnetic moments $\mu_{i}$ on $\mathrm{C}$ impurity versus the total magnetization of matrix atoms $\sum \mu^{\mathrm{NN}}$ in the $\mathrm{NN}$ of the impurity atom. The Fe-C results are from Ref. [19].

and $(\mathrm{Fe}+\mathrm{Mn})$ atoms in the $\mathrm{NN}$ of the $\mathrm{C}$ impurity. We observe a dispersion of supercells energies in both the Fe-Mn-C and $\mathrm{Fe}-\mathrm{C}$ alloys, which arises due to the presence of the various magnetic configurations in the $\mathrm{NN}$ of the interstitial impurity. For Fe-Mn-C alloys the distribution of total energies relative to $\sum \mu^{(\mathrm{Fe}+\mathrm{Mn})}[\mathrm{Fig} .5(\mathrm{~b})]$ is more uniform, while for the fcc $\mathrm{Fe}$ the minimum energies correspond to the supercells with the highest magnetic polarization of $\mathrm{Fe}$ atoms in the NN of impurity [see Fig. 5(a)]. A similar trend is observed in distribution of magnetic moments of the host atoms around the C impurity (see Fig. 3).

From the analysis of local and global effects of the $\mathrm{C}$ impurity on matrix properties we can see that the presence of manganese in alloy enlarges the volumes of voids in the crystal, which in turn weakens the deformation of $\mathrm{Fe}-\mathrm{C}$ bonds and reduces the screening radius of the impurity.
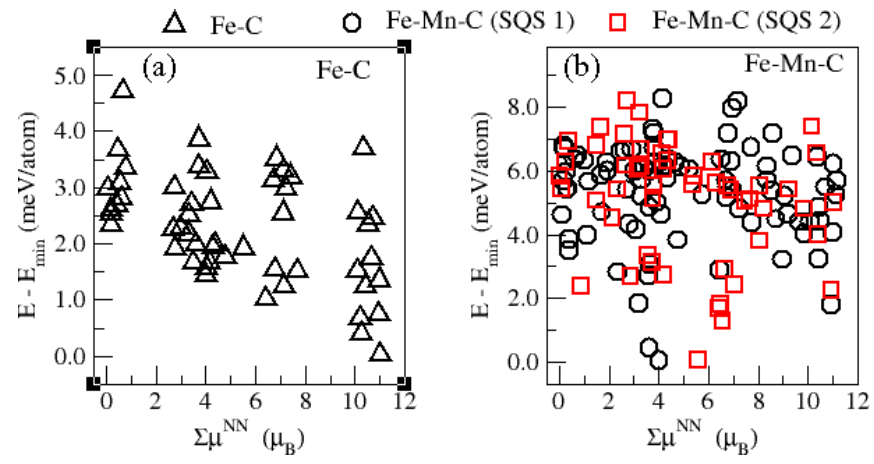

FIG. 5. Energies of MSM supercells with various impurity positions versus the total magnetic moment of matrix atoms $\sum \mu^{\mathrm{NN}}$ in the first CS of the C impurity for Fe-C (a) and Fe-Mn-C (b) alloys. Energies are calculated relative to the minimum energy $E_{\min }$ obtained for each SQS supercell. The Fe-C results were taken from Ref. [19].

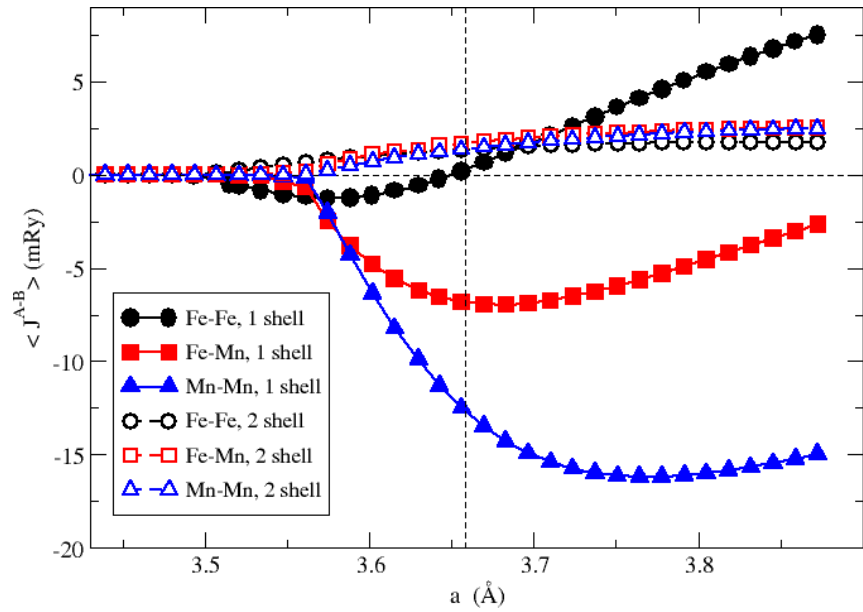

FIG. 6. Calculated average exchange pair interactions in paramagnetic Fe-Mn alloy plotted versus the lattice parameter.

\section{DISCUSSION}

For better understanding the features of impurity solution in Fe-Mn-C alloys, we plot the pair exchange interactions $J_{i j}$ of classical Heisenberg Hamiltonian as a function of lattice parameter (see in Fig. 6). The exchange interactions $J_{i j}$ were calculated for the host fcc $\mathrm{Fe}_{79.6} \mathrm{Mn}_{20.4}$ alloy using the Exact Muffin-Tin Orbitals method combined with CPA (EMTOCPA) [40-44].

As shown earlier in Ref. [19], the strong dependence of $J_{i j}$ on the lattice parameter might be considered as the possible explanation of existence of so-called SFT in the Fe-C alloys. The similar behavior was observed earlier in various magnetic configurations of $\gamma$-Fe: ferromagnetic [37], DLM [19], spin spirals [45]. According to our EMTO-CPA calculations of $\gamma$-Fe [19], the sign of the exchange interaction $J^{\mathrm{Fe}-\mathrm{Fe}}$ is negative for the range of lattice parameter from 3.50 to $3.63 \AA$. However, with further increasing of the lattice parameters $J^{\mathrm{Fe}-\mathrm{Fe}}$ becomes positive and rapidly increases. It is known that the stretching deformations around the impurity atom increase the distance between iron atoms in the first CS, which in turn changes the sign of $J^{\mathrm{Fe}-\mathrm{Fe}}$ exchange interaction. Therefore, the carbon impurity can change the sign of exchange interaction between $\mathrm{Fe}$ atoms in its first coordination sphere. Moreover, the carbon impurity stimulates the formation of partially or fully ferromagnetic local regions with lower solution enthalpy. In particular, according to Fig. 5(a), the lowest value of solution energy corresponds to the supercell realizations with the highest magnetic polarization of Fe atoms in the NN of carbon impurity.

In the manganese containing alloy, the additional Fe-Mn and $\mathrm{Mn}-\mathrm{Mn}$ pair interactions must also be considered. These interactions are sufficiently strong, and they remain negative for the range of lattice parameters close to equilibrium, as well as for larger lattice parameters corresponding to possible dilatation effects. Therefore, the ferromagnetic alignments of local moments on chemically nonequivalent atoms around the $\mathrm{C}$ impurity would not satisfy the signs of Fe-Mn and $\mathrm{Mn}-\mathrm{Mn}$ pair interactions. This is due to the local magnetic frustrations caused by the large negative values of $J^{\mathrm{Fe}-\mathrm{Mn}}$ and $J^{\mathrm{Mn}-\mathrm{Mn}}$ interactions. As a matter of fact, the frustrations also explain 

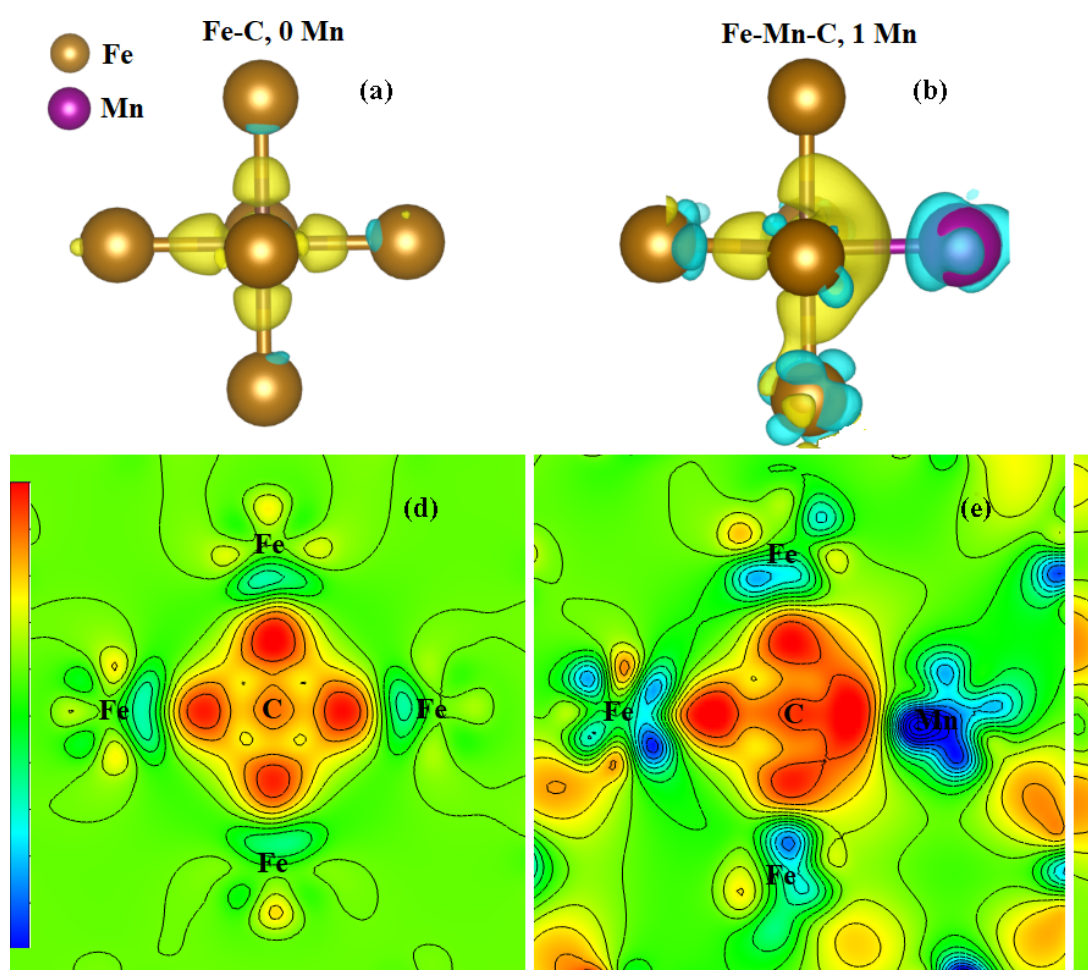
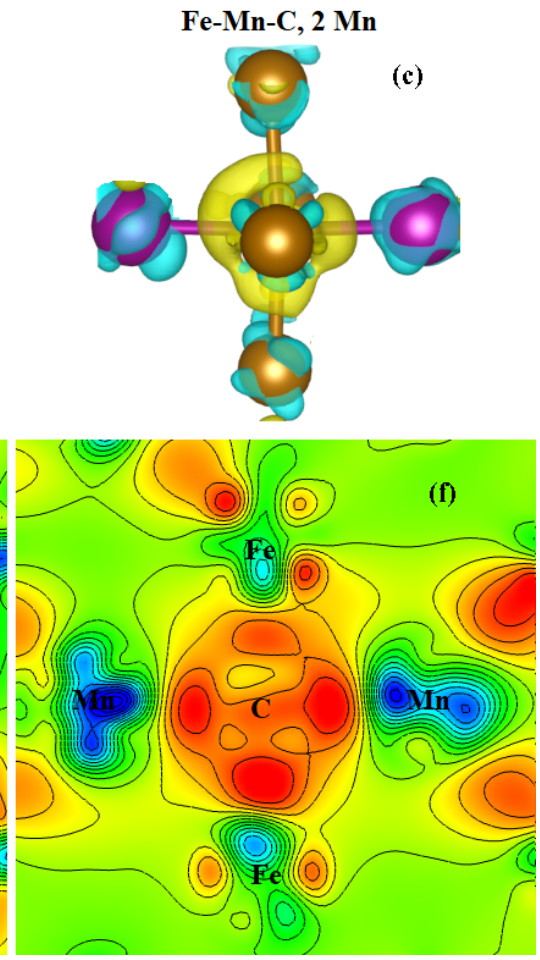

FIG. 7. 3D and 2D maps of charge density difference for Fe-C and Fe-Mn-C alloys plotted in the plane containing C impurity: (a) and (d) without $\mathrm{Mn}$ atoms (Fe-C, $0 \mathrm{Mn}$ ); (b) and (e) with 1 atom of $\mathrm{Mn}$ in the NN (Fe-Mn-C, $1 \mathrm{Mn}$ ); (c) and (f) with two atoms of Mn in NN (Fe-Mn-C, 2 Mn).

the existence of the magnetic moments with magnitudes from 0.0 to $0.5 \mu_{\mathrm{B}}$, because reducing and even quenching the local moments is a well-known way for a system to respond to the magnetic frustrations [46].

Now let us consider the map of charge density difference for $\mathrm{Fe}-\mathrm{C}$ and $\mathrm{Fe}-\mathrm{Mn}-\mathrm{C}$ alloys plotted in the plane containing the $\mathrm{C}$ impurity. We will discuss specific magnetic configurations of the paramagnetic state of the alloys. Figure 7 shows three different cases that correspond to different numbers of $\mathrm{Mn}$ atoms in the first CS of the $\mathrm{C}$ impurity. The analysis of the electron density difference reveals the redistribution of the electron density in the system due to the modification of chemical bonds. In binary $\mathrm{Fe}-\mathrm{C}$ alloys, the interatomic interaction between $\mathrm{Fe}$ and $\mathrm{C}$ lead to the transfer of the electronic density from the iron atom to the carbon atom. At the same time, one sees that the excessive electrons around the carbon atom are symmetrically localized near lines pointing towards each Fe atom.

In the regions between $\mathrm{Fe}$ atoms and close to carbon, the electronic density is depleted [see Figs. 7(a) and 7(d)]. A $\mathrm{Mn}$ atom in the first CS leads to the delocalization of the charge, and the region with excessive electron densities becomes broader. In the case of single $\mathrm{Mn}$ atom in the nearest neighborhood of carbon, the electron density forms excessive charge along the line that connects the centers of $\mathrm{Mn}$ and $\mathrm{C}$ atoms as well as along the line that connects $\mathrm{Mn}$ and $\mathrm{Fe}$ atoms [see Fig. 7(b)]. One can observe the regions with electron deficiency near the manganese site, while around the $\mathrm{Fe}$ atom in the first $\mathrm{CS}$ of the $\mathrm{C}$ impurity and in the second CS of Mn the electron density remains localized.
Note that the electronegativities of $\mathrm{C}, \mathrm{Fe}$ and $\mathrm{Mn}$ are equal to $2.55,1.83$ and 1.55, respectively. Because Mn has the lowest electronegativity, the charge transfer from manganese toward carbon and iron sites can indeed be expected. This is demonstrated in Fig. S1 (see the Supplemental Material), where the maps of charge density differences are plotted in the planes distant from the impurity. One of these planes contains only Fe atoms (Supplemental Material, Fig. S1, left panel), while the other one contains both, Fe and $\mathrm{Mn}$ atoms (Supplemental Material, Fig. S1, right panel). From Fig. S1 we see that the electron density is transferred toward the more electronegative $\mathrm{Fe}$ atom. Moreover, the electron density between $\mathrm{Fe}$ and $\mathrm{Mn}$ atoms is higher compared to $\mathrm{Fe}-\mathrm{Fe}$ and Mn-Mn pairs.

In the case of two $\mathrm{Mn}$ atoms in the first $\mathrm{CS}$ of the $\mathrm{C}$ impurity, the region with excessive electron densities becomes broader and covers almost the whole spatial sphere around the carbon. The screening of the impurity becomes more effective despite strong local dilatations in the first CS (see Fig. 1). Lattice deformation sufficiently weakens for both Fe and Mn; and becomes symmetric already in the second CS. Thus, the presence of $\mathrm{Mn}$ in the first CS of impurity induces charge delocalization (in comparison to pure fcc Fe), which in turn provides the effective screening of the $\mathrm{C}$ impurity.

\section{CONCLUSIONS}

In the present work, the solution enthalpy of carbon impurity into the paramagnetic Fe-Mn alloys has been calculated within the framework of DFT. Calculations have been carried 
out within the generalization of the model that describes the point defects in paramagnetic hosts towards the case of alloy hosts. The model allows us to account for thermal magnetic fluctuations in the paramagnetic chemically disordered matrix with point defects.

According to theoretical results, in the alloys with Mn concentration $\sim 20$ at. $\%$, the solution energy of carbon impurity becomes lower compared to the pure paramagnetic $\gamma$ iron. This behavior of the solution energy qualitatively agrees with the experiment. The origin of the reduction of the solution enthalpy can be traced to following reasons:

(i) Alloying with $\mathrm{Mn}$ increases the lattice parameter. This, in turn, decreases the distortions of Fe-C bonds, and increases the spatial size of the octahedral sites where the carbon impurity is dissolved. (ii) Analysis of charge density maps shows the delocalization of electron density in the first CS of the C impurity, in the presence of manganese atoms. This makes the screening of the impurity more efficient and weakens the lattice deformation starting from the second CS.

\section{ACKNOWLEDGMENTS}

Theoretical calculations were supported by the Russian Science Foundation (Project No. 18-12-00492). Financial support from the Swedish Research Council (VR) through Grant No. 2015-04391 and the Swedish Government Strategic Research Area in Materials Science on Functional Materials at Linköping University (Faculty Grant SFO-Mat-LiU No. 2009-00971) is gratefully acknowledged.
[1] M. Ghasri-Khouzani and J. R. McDermit, Mater. Sci. Eng. A 621, 118, (2015).

[2] H. W. King and M. A. Peters, Can. Metall. Q. 36, 137 (1997).

[3] F. Trichter, A. Rabinkin, M. Ron, and A. Sharfstein, Scr. Metall. 12, 431 (1978).

[4] T. Hickel, S. Sandlöbes, R. K. W. Marceau, A. Dick, I. Bleskov, J. Neugebauer, and D. Raabe, Acta Mater. 75, 147 (2014).

[5] K. R. Limmer, J. E. Medvedeva, D. C. Van Aken, and N. I. Medvedeva, Comput. Mater. Sci. 99, 253 (2015).

[6] N. I. Medvedeva, M. S. Park, D. C. Van Aken, and J. E. Medvedeva, J. Alloys Compd. 582, 475 (2014).

[7] T. Gebhardt, D. Music, B. Hallstedt, M. Ekholm, I. A. Abrikosov, L. Vitos, and J. M. Schneider, J. Phys.: Condens. Matter 22, 295402 (2010).

[8] T. Hickel, A. Dick, B. Grabowski, F. Körmann, and J. Neugebauer, Steel Res. Int. 80, 4 (2009).

[9] A. Georges, G. Kotliar W. Krauth, and M. Rozenberg, Rev. Mod. Phys. 68, 13 (1996).

[10] J. Hubbard, Phys. Rev. B 19, 2626 (1979).

[11] J. Hubbard, Phys. Rev. B 20, 4584 (1979).

[12] J. Hubbard, Phys. Rev. B 23, 5974 (1981).

[13] H. Hasegawa, J. Phys. Soc. Jpn. 46, 1504 (1979).

[14] H. Hasegawa, J. Phys. Soc. Jpn. 49, 178 (1980).

[15] B. L. Gyorffy, A. J. Pindor, J. Staunton, G. M. Stocks, and H. Winter, J. Phys. F 15, 1337 (1985).

[16] B. Alling, T. Marten, and I. A. Abrikosov, Phys. Rev. B 82, 184430, (2010).

[17] A. Zunger, S.-H. Wei, L. G. Ferreira, and J. E. Bernard, Phys. Rev. Lett. 65, 353 (1990).

[18] P. Steneteg, B. Alling, and I. A. Abrikosov, Phys. Rev. B 85, 144404 (2012).

[19] A. V. Ponomareva, Yu. N. Gornostyrev, and I. A. Abrikosov, Phys. Rev. B 90, 014439 (2014).

[20] G. Kresse and J. Furthmüller, Comput. Mater. Sci. 6, 15 (1996).

[21] G. Kresse and J. Furthmüller, Phys. Rev. B 54, 11169 (1996).

[22] G. Kresse and D. Joubert, Phys. Rev. B 59, 1758 (1999).

[23] P. E. Blöchl, Phys. Rev. B 50, 17953 (1994).

[24] J. P. Perdew, K. Burke, and M. Ernzerhof, Phys. Rev. Lett. 77, 3865 (1996).
[25] H. Hellmann, Einführung in die Quantumchemie (Deuticke, Leipzig, 1937).

[26] H. J. Monkhorst and J. D. Pack, Phys. Rev. B 13, 5188 (1976).

[27] See Supplemental Material at http://link.aps.org/supplemental/ 10.1103/PhysRevMaterials.4.024401 for information on chosen lattice parameters of Fe-Mn alloys.

[28] N. Shulumba, B. Alling, O. Hellman, E. Mozafari, P. Steneteg, M. Oden, and I. A. Abrikosov, Phys. Rev. B 89, 174108 (2014).

[29] I. Stockem, A. Bergman, A. Glensk, T. Hickel, F. Körmann, B. Grabowski, J. Neugebauer, and B. Alling, Phys. Rev. Lett. 121, 125902 (2018).

[30] A. Ludstech, Acta Cryst. A 28, 59 (1972).

[31] M. Hillert and M. Jarl, Metall. Trans. A 6, 553 (1975).

[32] J. A. Lobo and G. H. Geiger, Metall. Trans. A 7, 1359 (1976).

[33] R. Ferro and A. Saccone, Intermetallic Chemistry, Pergamon Materials Science (Elsevier, Amsterdam, 2008).

[34] D. Bouchard and J. S. Kirkaldy, J. Alloys Compd. 283, 311 (1999).

[35] T. Wada, H. Wada, J. F. Elliott, and J. S. Chipman, Metall. Trans. 31657 (1972).

[36] B. Drittler, M. Weinert, R. Zeller, and P. H. Dederichs, Phys. Rev. B 39, 930 (1989).

[37] A.V. Ruban, M. I. Katsnelson, W. Olovsson, S. I. Simak, and I. A. Abrikosov, Phys. Rev. B 71, 054402 (2005).

[38] Landolt-Börnstein, in Magnetic Properties of Metals: $3 d, 4 d$, and $5 d$ Elements, Alloys and Compounds, New Series, Group III, edited by K. Kanematsu, S. Misawa, M. Shiga, H. Wada, and H. P. J. Wijn (Springer-Verlag, Berlin, 1997), Vol. 32A.

[39] H.-Ch. Mertins, S. Valencia, W. Gudat, P. M. Oppeneer, O. Zaharko, and H. Grimmer, Europhys. Lett. 66, 743 (2004).

[40] L. Vitos, Phys. Rev. B 64, 014107 (2001).

[41] L. Vitos, I. A. Abrikosov, and B. Johansson, Phys. Rev. Lett. 87, 156401 (2001).

[42] P. Soven, Phys. Rev. 156, 809 (1967).

[43] B. L. Gyorffy, Phys. Rev. B 5, 2382 (1972).

[44] J. S. Faulkner, Prog. Mater. Sci. 27, 1 (1982).

[45] S. V. Okatov, Yu. N. Gornostyrev, A. I. Lichtenstein, and M. I. Katsnelson, Phys, Rev. B 84, 214422 (2011).

[46] P. A. Korzhavyi, A. V. Ruban, J. Odqvist, J.-O. Nilsson, and B. Johansson, Phys, Rev. B 79, 054202 (2009). 